\title{
The effect of spontaneous breathing on systemic interleukin-6 during ventilator
} weaning

\author{
J. Sellarés*,\# , H. Loureiro*,\#, M. Ferrer*,", R. Amaro*, R. Farré",, and A. Torres*,\#
}

ABSTRACT: During the weaning process, spontaneous breathing trials (SBTs) involve cardiopulmonary stress for ventilated patients. As interleukin (IL)-6 is a major modulator of the stress response, we hypothesised that systemic IL-6 increases during a SBT and that this increase is more evident in SBT failure.

49 SBTs of 30 -min duration were performed on different mechanically ventilated patients, and classified as SBT failure or success. Blood samples were drawn before and at the end of the SBT. An additional sample was drawn $24 \mathrm{~h}$ later in a subset of patients $(n=39)$. Serum IL-6 levels and other inflammatory mediators commonly associated with stress were determined.

IL-6 levels increased from mechanical ventilation to spontaneous breathing in all patients $(p=0.02)$ and in the chronic obstructive pulmonary disease (COPD) population $(p=0.05)$ with SBT failure compared with success, but not in non-COPD patients $(p=0.12)$. After $24 \mathrm{~h}$ of SBT stress, IL-6 levels decreased in patients with SBT failure (under mechanical ventilation at that point) $(p=0.02)$ and those with weaning success $(p=0.04)$. No changes were observed in the remaining inflammatory mediators.

Systemic IL-6 increases during a 30-min, failed SBT, especially in COPD patients. Future studies may corroborate the different IL-6 responses among different populations who initiate weaning, together with the potential clinical implications.

KEYWORDS: Chronic obstructive pulmonary disease, critical care, interleukin-6, mechanical ventilation, spontaneous breathing trial, weaning

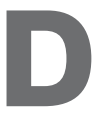
uring the weaning process, the routine employment of daily spontaneous breathing trials (SBTs) or the use of low levels of pressure support as a weaning method decreases the duration of ventilation support and improves survival [1-4]. However, this test could result in cardiopulmonary stress for some patients. Stress is defined as "the state to which an organism is led because of external or internal forces (stressors) that threaten to alter its dynamic equilibrium (homeostasis)" [5]. This definition of stress could be applied to the weaning process after close examination of the factors that influence the pathophysiology of weaning failure. The transition from mechanical ventilation to spontaneous breathing entails an increase in respiratory muscle energy demands to cope with respiratory load (respiratory stress) [6], with a consequent increase in oxygen demand $[7,8]$, which in turn requires an increase in cardiac output (cardiovascular stress) [7]. Although all patients who perform a SBT must face this stress, patients who fail the SBT are subjected to higher pulmonary [6] and cardiovascular stress
$[7,9,10]$ than those who tolerate the trial. If we assume that during a failed SBT, patients experience high cardiopulmonary stress, we could also assume that the different biological systems associated with stress response could be also activated during this process [5].

When the stress system is activated, there is a consequent increase in plasma catecholamines due to sympathetic stimulation [11]. The secretion of catecholamines during stress is associated with systemic interleukin (IL)-6 production [12]. Considering SBT as a "stress model", we hypothesised that, after the patient has been subjected to mechanical ventilation, the "stress" of initiating spontaneous breathing is associated with an increase in systemic IL-6. As IL-6 production seems to be related to the intensity of stress in other conditions (especially in acute exercise) $[13,14]$, we also hypothesised that the increase of IL- 6 during SBT is higher in patients who fail the trial, because this population typically develops higher cardiopulmonary stress. Although the primary focus of

\section{AFFILIATIONS}

*Respiratory Intensive Care Unit, Hospital Clínic-August Pi i Sunyer Biomedical Research Institute (IDIBAPS),

"Unitat Biofisica i Bioenginyeria, Facultat Medicina, Universitat Barcelona-IDIBAPS, Barcelona, and \#Centro de Investigación Biomédica En Red - Enfermedades Respiratorias (CB06/06/0028), Barcelona, Spain.

\section{CORRESPONDENCE}

J. Sellarés

Servei de Pneumologia

Hospital Clinic

Villarroel 170

08036 Barcelona

Spain

E-mail: sellares@clinic.ub.es

Received:

Feb 282011

Accepted after revision:

July 012011

First published online:

July 202011

European Respiratory Journal

Print ISSN 0903-1936

Online ISSN 1399-3003 
our study was IL-6, we also assessed tumour necrosis factor (TNF)- $\alpha$, IL-1 $\beta$, IL-8, IL-10 and C-reactive protein (CRP) to determine the inflammatory pattern associated with IL-6 changes, since these markers are usually related to IL-6 response under different stress conditions [5, 14].

\section{METHODS AND MATERIALS Patients}

This prospective study was conducted from November 2005 to June 2009 in a respiratory intensive care unit at the Hospital Clinic of Barcelona, Spain. All intubated and mechanically ventilated patients $(\geqslant 48 \mathrm{~h}$ ) were consecutively included for the study when the following criteria were fulfilled: 1) improvement or resolution of the underlying causes of acute respiratory failure; 2) no fever $\left(\geqslant 38^{\circ} \mathrm{C}\right)$ or hypothermia $\left(<36^{\circ} \mathrm{C}\right)$; 3 ) haemoglobin $\geqslant 9 \mathrm{~g} \cdot \mathrm{dL}^{-1}$; 4) absence of vasoactive drugs; 5) normal consciousness after discontinuing sedation; and 6) arterial oxygen tension $\left(\mathrm{Pa}_{1} \mathrm{O}_{2}\right)>60 \mathrm{mmHg}$ with inspiratory oxygen fraction $\left(\mathrm{FI}, \mathrm{O}_{2}\right) \leqslant 0.4$ and positive end-expiratory pressure $\leqslant 5 \mathrm{cmH}_{2} \mathrm{O}$. Exclusion criteria were: 1) tracheotomy or other upper airway disorders; 2 ) active upper gastrointestinal bleeding; 3) lack of cooperation; and 4) decision to limit lifesustaining treatments. The study was approved by the ethics committee of the Hospital Clinic of Barcelona and informed written consent was obtained in all cases.

\section{Protocol}

A T-piece trial was performed in all eligible patients. Endotracheal suctioning was performed before the study. SBT failure was defined as the presence and persistence of one of the following criteria: 1) respiratory frequency ( $f R$ ) $>35$ breath$\left.\mathrm{s} \cdot \mathrm{min}^{-1} ; 2\right)$ arterial oxygen saturation measured by pulse oximetry $<90 \%\left(80 \%\right.$ in chronic respiratory failure) at $\left.\mathrm{FI}_{1} \mathrm{O}_{2} \geqslant 0.4 ; 3\right)$ heart rate $>140$ or $<50$ beats $\cdot \mathrm{min}^{-1}$, or increases or decreases of $>20 \%$ compared with mechanical ventilation; 4 ) systolic blood pressure $>180$ or $<70 \mathrm{mmHg}$, or increases or decreases of $>20 \%$ compared with mechanical ventilation; 5) decreased consciousness, agitation or diaphoresis; and 6) thoracoabdominal paradoxical movement $[15,16]$. If no signs of SBT failure appeared in $30 \mathrm{~min}$, the trial was considered successful (SBT success) and patients were extubated. Alternatively, if signs of failure appeared during this period, patients were reconnected to the ventilator (SBT failure). The decision whether to extubate the patient or reinstitute mechanical ventilation was made solely by the primary physician.

\section{Data collection and definitions}

All relevant data from patients' medical records and bedside flow charts were reviewed at entry and at the end of the protocol. Maximal inspiratory pressure (MIP) and maximum expiratory pressure (MEP) were measured prior to the SBT, using a unidirectional valve [17].

During the first $48 \mathrm{~h}$ after extubation, the patients with SBT success were followed up and classified as follows. Weaning success was defined as extubation and the absence of ventilatory support $48 \mathrm{~h}$ following the extubation [2]. Respiratory failure after extubation was defined as, in addition to re-intubation criteria, the presence and persistence, within $48 \mathrm{~h}$ after extubation, of at least two of the following: 1) respiratory acidosis (arterial $\mathrm{pH}<7.35$ with arterial carbon dioxide tension $>45 \mathrm{mmHg}$ ); 2) arterial oxygen saturation measured by pulse oximetry $<90 \%$ or $\mathrm{Pa}_{2} \mathrm{O}_{2}$
$<60 \mathrm{mmHg}$ at $\mathrm{FI}, \mathrm{O}_{2} \geqslant 0.5$; 3) $\mathrm{fR}>35$ breaths $\cdot \mathrm{min}^{-1}$; 4) decreased consciousness, agitation or diaphoresis; and 5) clinical signs suggestive of respiratory muscle fatigue and/or increased work of breathing, such as the use of respiratory accessory muscles, paradoxical motion of the abdomen or retraction of the intercostal spaces [16]. Immediate re-intubation criteria were pre-defined as any of the following major clinical events: respiratory or cardiac arrest; respiratory pauses with loss of consciousness or gasping for air; psychomotor agitation inadequately controlled by sedation; massive aspiration; persistent inability to remove respiratory secretions; heart rate $<50$ beats $\cdot \mathrm{min}^{-1}$ with loss of alertness; and severe haemodynamic instability without response to fluids and vasoactive drugs [16].

The causes of respiratory failure after extubation were assigned using adapted published definitions: 1) upper airway obstruction; 2) aspiration or excess respiratory secretions; 3 ) congestive heart failure; 4) respiratory failure; and 5) encephalopathy [18]. Rescue therapy with noninvasive ventilation (NIV) was used in the case of respiratory failure after extubation if immediate reintubation was not necessary [16].

\section{Inflammatory response}

Venous blood samples were collected during mechanical ventilation, just before beginning the protocol and at the end of the SBT. In order to assess the recovery response of inflammatory mediators after the SBT, an additional sample was obtained $24 \mathrm{~h}$ after the protocol in a subset of patients $(n=39)$; it was not possible to collect the sample in the remaining patients. Serum was obtained after centrifuging the sample at $300 \times g(1,800 \mathrm{rpm})$ for $10 \mathrm{~min}$, and it was then frozen at $-80^{\circ} \mathrm{C}$ until further processing. The determination of cytokine levels was performed with a commercial enzyme immunoassay technique (Biosource, Nivelles, Belgium). The limits of detection were as follows. IL- $1 \beta$ and IL-6: $2 \mathrm{pg} \cdot \mathrm{mL}^{-1}$; TNF- $\alpha$ : $3 \mathrm{pg} \cdot \mathrm{mL}^{-1}$; IL-8: $0.7 \mathrm{pg} \cdot \mathrm{mL}^{-1}$; and IL-10: $1 \mathrm{pg} \cdot \mathrm{mL}^{-1}$. CRP was measured with an immunoturbidimetric method using a commercially available test (Bayer, Leverkusen, Germany) with an Advia 2400 (Siemens AG, Munich, Germany).

\section{Statistical analysis}

Data are presented as mean $\pm \mathrm{SD}$, except variables that were not normally distributed, which are presented as median (interquartile range (IQR)). Absolute changes of serum inflammatory mediators levels expressed as the change between mechanical ventilation and the end of the SBT were calculated, and included in the analysis. All data were tested for normality using Kolmogorov-Smirnov test. All inflammatory mediators were not normally distributed except CRP. Levels that were undetectable were assigned a value equal to the lower limit of detection for the assay. In order to correctly assess the inflammatory response associated with SBT, we performed data analysis of inflammatory markers separately in two stages: from mechanical ventilation to SBT (inflammatory response over the SBT) and from SBT to $24 \mathrm{~h}$ after the trial (recovery phase after SBT). Comparisons were carried out by unpaired or paired t-test when appropriate, if the data were normally distributed. Otherwise, Wilcoxon and Mann-Whitney nonparametric tests were used for paired and unpaired comparisons, respectively.

Because chronic obstructive pulmonary disease (COPD) appears to be an independent risk factor for increased duration 
of weaning and weaning failure [19], we performed separate post hoc analyses comparing SBT failure and success in this population, and in patients without COPD.

In the absence of previous data concerning IL-6 changes during weaning, we initiated a study analysing IL- 6 changes during weaning in 26 patients [20]. In this sample, we had already observed that: 1) IL-6 increased in patients who failed the SBT; and 2) when we divided the population in COPD and nonCOPD patients, the increase was only present in COPD patients who failed the SBT. However, the number of patients in each subgroup was limited, so we performed a power analysis. We observed an increase of $29 \mathrm{pg} \cdot \mathrm{mL}^{-1}$ in the subgroup of COPD patients who failed the SBT. Assuming a similar result in the non-COPD patients who failed the SBT (standard deviation $28 \mathrm{pg} \cdot \mathrm{mL}^{-1}$ ), the sample size calculation revealed a minimum of eight patients (power $80 \%$; two-sided $\alpha=0.05$ ).

The level of significance was set at 0.05 in all tests (all twotailed). Statistical analysis was performed with SPSS 16 (SPSS, Chicago, IL, USA).

\section{RESULTS}

\section{Physiological characteristics}

General clinical characteristics of the 49 patients included in this study are summarised in table 1. Of the 49 SBTs assessed in this population, $33(67 \%)$ were considered SBT success and $16(33 \%)$ SBT failure. Within the success group, seven patients developed respiratory failure after extubation and 26 were classified as weaning success. The causes of failure after extubation were respiratory failure $(n=6)$, where NIV was initiated, and encephalopathy $(n=1)$, where it was decided to limit life support therapy.

The physiological parameters of all patients and the subgroup of the COPD population are shown in table 2 . In all trials, $f R$ and mean systemic arterial pressure significantly increased at the end of the SBT in failure and success groups; heart rate increased significantly in the SBT failure group but not in the SBT success group. There was also a significant decrease of $\mathrm{pH}$ and $\mathrm{Pa}_{\mathrm{a}_{2}} \mathrm{O}_{2}$ in all trials. The MIP, MEP and $f R /$ total volume ratio did not discriminate between patients with SBT success and failure in all patients $(p=0.16, p=0.81$ and $p=0.11$, respectively) and in the COPD subgroup $(\mathrm{p}=0.56, \mathrm{p}=0.57$ and $\mathrm{p}=0.22$, respectively).

\section{Inflammatory response over the SBT in the population as a whole}

In the 49 patients in the study, during mechanical ventilation, baseline levels of IL-6 were not significantly different when patients were divided into SBT failure or success groups $(p=0.12)$. Over the SBT, the increase in IL-6 was only statistically significant in patients with failure $(\mathrm{p}=0.02)$ (fig. 1a). Change in $(\Delta)$ IL-6 in SBT failure was significantly increased compared with the success group $(p=0.05)$ (fig. $1 c)$. For the remaining mediators, no differences were observed in baseline values and no significant changes were observed during the SBT (table 3).

\section{Inflammatory response over the SBT in COPD patients}

23 weaning trials were assessed in these patients. No significant differences were observed in median (IQR) baseline levels of IL- 6 between the failure $(n=8)$ and success $(n=15)$ groups: 49 (22-147) $\mathrm{pg} \cdot \mathrm{mL}^{-1}$ in SBT failure and $35(11-40) \mathrm{pg} \cdot \mathrm{mL}^{-1}$ in SBT

\begin{tabular}{lc} 
TABLE 1 Baseline characteristics of patients on entry into \\
study \\
Age yrs & $68 \pm 13$ \\
Males/females & $41 / 8$ \\
Mechanical ventilation time days & $7 \pm 5$ \\
APACHE II score & \\
Admission & $17 \pm 7$ \\
Study entry & $10 \pm 4$ \\
Underlying diseases & \\
Chronic respiratory disorders ${ }^{*}$ & $33(67)$ \\
$\quad$ COPD & 23 \\
$\quad$ Non-COPD & 10 \\
Chronic heart disorders & $13(26)$ \\
Immunosuppression ${ }^{+}$ & $7(14)$ \\
Diabetes mellitus & $12(24)$ \\
Reason for initiating mechanical ventilation & \\
Exacerbation of chronic respiratory disorders & $13(27)$ \\
Congestive heart failure & $6(12)$ \\
Pneumonia & $6(12)$ \\
Sepsis & $5(10)$ \\
Post-operative respiratory failure & $2(4)$ \\
Neurological disease & $2(4)$ \\
Other causes & $15(31)$ \\
\hline
\end{tabular}

Data are presented as mean $\pm S D, n$ or $n(\%)$, unless otherwise stated. $n=49$. APACHE: Acute Physiology and Chronic Health Evaluation; COPD: chronic obstructive pulmonary disease. ${ }^{\#}$ : including COPD, chronic bronchitis associated with dyspnoea and current or former history of smoking in the absence of pulmonary function testing, sequelae of pulmonary tuberculosis chest wall deformity or obesity associated with a restrictive ventilatory disorder, and bronchiectasis; ? including coronary artery disease, hypertensive or valvular heart diseases, and dilated myocardial disease of any cause; ${ }^{+}$: including neutropenia after chemotherapy or bone marrow transplantation, drug-induced immunosuppression in solid-organ transplantation, or as a result of corticosteroid or cytotoxic therapy, and HIV-related disorders.

success $(p=0.44)$. While IL-6 increased during the SBT in all patients who failed the weaning attempt $(p=0.05$; fig. $2 a)$, these changes were not observed in those who tolerated the SBT ( $p=0.64$; fig. $2 b$ ). In addition, $\Delta \mathrm{IL}-6$ in SBT failure was significantly higher $(\mathrm{p}=0.03)$ compared with the success group (fig. 2c). In contrast, patients without COPD (fig. 3) showed no significant changes during the SBT in failed $(n=8)$ and successful $(n=18)$ trials. No changes were observed in the remaining mediators.

\section{Inflammatory response $\mathbf{2 4} \boldsymbol{h}$ after the SBT}

In the 39 trials subgroup from which an additional sample was obtained $24 \mathrm{~h}$ after SBT, eight were classified as SBT failure, seven as respiratory failure after extubation and 24 as weaning success. In SBT failure (under mechanical ventilation at $24 \mathrm{~h}$ ), median (IQR) IL-6 levels decreased after $24 \mathrm{~h}$ of the trial from 82 (35-160) to $58(25-125) \mathrm{pg} \cdot \mathrm{mL}^{-1}(\mathrm{p}=0.02)$. In weaning success, IL-6 significantly decreased at $24 \mathrm{~h}$ after extubation from 42 (13-100) to $29(8-58) \mathrm{pg} \cdot \mathrm{mL}^{-1}(\mathrm{p}=0.04)$. In the respiratory failure after extubation group, IL-6 did not significantly change in $24 \mathrm{~h}$ (patients were breathing spontaneously when the sample was collected). No significant changes were observed in the remaining inflammatory markers. 
TABLE 2 Physiological parameters during mechanical ventilation (MV) and the spontaneous breathing trial (SBT) among patients with SBT failure or success

\begin{tabular}{|c|c|c|c|c|c|c|}
\hline & \multicolumn{3}{|c|}{ All trials ${ }^{\#}$} & \multicolumn{3}{|c|}{ COPD trials } \\
\hline & MV & SBT & p-value ${ }^{+}$ & MV & SBT & p-value ${ }^{+}$ \\
\hline SBT failure & $17 \pm 6$ & $24 \pm 11$ & 0.009 & $17 \pm 7$ & $20 \pm 9$ & 0.11 \\
\hline SBT success & $17 \pm 4$ & $20 \pm 5$ & 0.002 & $17 \pm 4$ & $19 \pm 4$ & 0.07 \\
\hline \multicolumn{7}{|c|}{ Heart rate beats $\cdot \min ^{-1}$} \\
\hline SBT success & $88 \pm 19$ & $79 \pm 32$ & 0.18 & $89 \pm 18$ & $90 \pm 32$ & 0.71 \\
\hline \multicolumn{7}{|l|}{$\bar{P}_{\text {sa }} \mathrm{mmHg}$} \\
\hline SBT failure & $84 \pm 13$ & $99 \pm 16$ & $<0.001$ & $87 \pm 15$ & $101 \pm 16$ & 0.002 \\
\hline SBT success & $86 \pm 15$ & $98 \pm 17$ & $<0.001$ & $89 \pm 12$ & $100 \pm 17$ & 0.001 \\
\hline \multicolumn{7}{|l|}{${\mathrm{Pa}, \mathrm{CO}_{2} \mathrm{mmHg}}$} \\
\hline SBT failure & $46 \pm 11$ & $50 \pm 14$ & 0.13 & $50 \pm 12$ & $56 \pm 16$ & 0.24 \\
\hline \multicolumn{7}{|l|}{$\mathrm{Pa}_{\mathrm{a}} \mathrm{O}_{2} \mathrm{mmHg}$} \\
\hline SBT failure & $91 \pm 19$ & $83 \pm 26$ & 0.07 & $93 \pm 18$ & $76 \pm 20$ & 0.05 \\
\hline SBT success & $101 \pm 19$ & $92 \pm 26$ & 0.01 & $102 \pm 24$ & $92 \pm 32$ & 0.05 \\
\hline \multicolumn{7}{|l|}{$f_{\mathrm{R}} / V_{\mathrm{T}}$} \\
\hline SBT failure & & $100 \pm 70$ & & & $58 \pm 27$ & \\
\hline SBT success & & $73 \pm 40$ & & & $77 \pm 35$ & \\
\hline \multicolumn{7}{|l|}{ MIP $\mathrm{cmH}_{2} \mathrm{O}$} \\
\hline SBT failure & & $42 \pm 18$ & & & $51 \pm 21$ & \\
\hline SBT success & & $52 \pm 19$ & & & $44 \pm 13$ & \\
\hline \multicolumn{7}{|l|}{ MEP $\mathrm{cmH}_{2} \mathrm{O}$} \\
\hline SBT failure & & $66 \pm 29$ & & & $78 \pm 28$ & \\
\hline
\end{tabular}

Data are presented as mean $\pm \mathrm{SD}$, unless otherwise stated. COPD: chronic obstructive pulmonary disease; fR: respiratory frequency; $\bar{P}_{\text {sa: }}$ mean systemic arterial pressure $\mathrm{Pa}_{1} \mathrm{CO}_{2}$ : arterial carbon dioxide tension; $\mathrm{Pa}, \mathrm{O}_{2}$ : arterial oxygen tension; $\mathrm{V} \mathrm{T}$ : tidal volume; MIP: maximal inspiratory pressure; $\mathrm{MEP}:$ maximal expiratory pressure. ${ }^{\#}$ : $\mathrm{n}=49$; $\because \mathrm{n}=23{ }^{+}{ }^{+}$: paired comparisons between MV and SBT in each group.

\section{DISCUSSION}

The main findings of the study are that in ventilated patients who performed a 30-min SBT: 1) IL-6 increase was higher in SBT failure compared with success; 2) when patients were divided in COPD and non-COPD subgroups, only the subset of patients with COPD increased IL-6 during the SBT; 3) at $24 \mathrm{~h}$ after SBT, IL-6 decreased in patients who remained ventilated (SBT failure) or in weaning success; and 4) the remaining inflammatory mediators assessed did not significantly change during weaning.
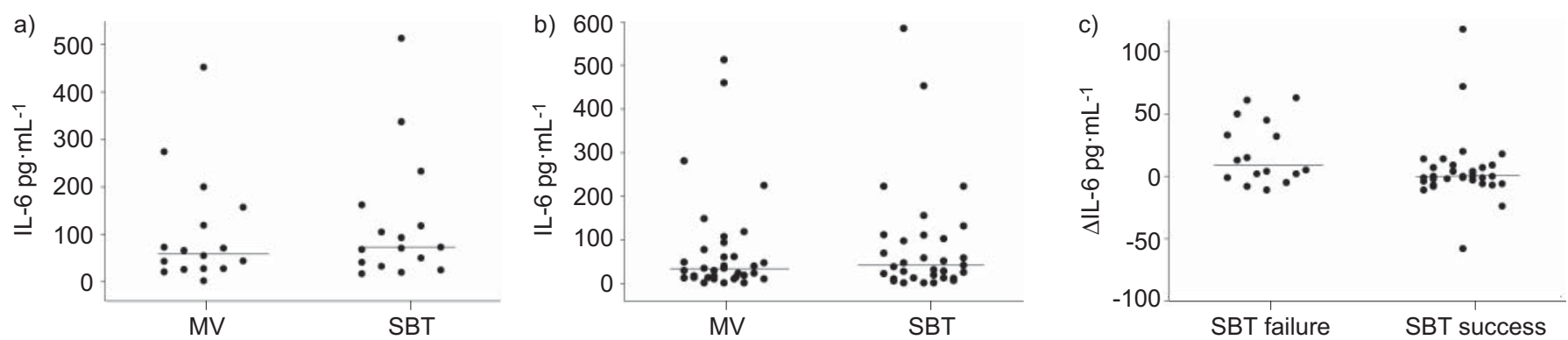

FIGURE 1. Individual values of interleukin (IL)-6 concentration during mechanical ventilation (MV) and at the end of a spontaneous breathing trial (SBT) in patients with a) SBT failure $(p=0.02)$ and b) SBT success $(p=0.46)$, and $c)$ the absolute change in $(\Delta)$ IL-6 between MV and SBT, comparing SBT failure and success in the whole study population $(p=0.05)$. - : median. 


\begin{tabular}{|c|c|c|c|}
\hline \multirow[t]{2}{*}{ TABLE 3} & \multicolumn{3}{|c|}{$\begin{array}{l}\text { Concentrations of cytokines and C-reactive } \\
\text { protein (CRP) during mechanical ventilation (MV) } \\
\text { and the spontaneous breathing trial (SBT) in the } \\
\text { overall population }\end{array}$} \\
\hline & MV & SBT & p-value ${ }^{\#}$ \\
\hline \multicolumn{4}{|l|}{$\mathrm{IL}-1 \beta \mathrm{pg} \cdot \mathrm{mL}^{-1}$} \\
\hline SBT failure & $2(0-3)$ & $2(1-3)$ & 0.71 \\
\hline SBT success & $2(0-2)$ & $2(0-3)$ & 0.59 \\
\hline \multicolumn{4}{|l|}{$\mathrm{IL}-6 \mathrm{pg} \cdot \mathrm{mL}^{-1}$} \\
\hline SBT failure & $60(28-147)$ & $72(35-151)$ & 0.02 \\
\hline SBT success & 35 (15-86) & $40(13-105)$ & 0.46 \\
\hline \multicolumn{4}{|l|}{ IL-8 $\mathrm{pg} \cdot \mathrm{mL}^{-1}$} \\
\hline SBT failure & 31 (11-95) & $26(12-63)$ & 0.71 \\
\hline SBT success & 38 (16-69) & $33(10-62)$ & 0.50 \\
\hline \multicolumn{4}{|l|}{$\mathrm{IL}-10^{\circ} \mathrm{pg} \cdot \mathrm{mL}^{-1}$} \\
\hline SBT failure & $1(1-3)$ & $1(1-4)$ & 0.88 \\
\hline SBT success & $1(1-4)$ & $1(1-3)$ & 0.25 \\
\hline \multicolumn{4}{|l|}{ TNF- $\alpha$ pg $\cdot \mathrm{mL}^{-1}$} \\
\hline SBT failure & $12(6-25)$ & $15(5-24)$ & 0.50 \\
\hline SBT success & $8(4-17)$ & $8(4-15)$ & 0.83 \\
\hline \multicolumn{4}{|l|}{ CRP $\mathrm{mg} \cdot \mathrm{dL}^{-1}$} \\
\hline SBT failure & $10 \pm 8$ & $10 \pm 9$ & 0.30 \\
\hline SBT success & $7 \pm 7$ & $7 \pm 6$ & 0.97 \\
\hline
\end{tabular}

To the best of our knowledge, the role of inflammatory markers during the weaning process has not been studied before. In order to explain the results obtained, we have to view weaning, particularly the SBT, as a test that could represent a cause of cardiopulmonary stress for the ventilated patient. KOKSAL et al. [21] compared the endocrine stress response developed during three methods of weaning using a T-tube, continuous positive airway pressure and pressure support in 60 patients who were successfully weaned. The patients of the T-tube group developed a greater increase in plasma insulin, cortisol, glucose and urine valine mandelic acid, showing a higher endocrine stress response than in the other weaning modes. Along the same lines, sympathoadrenal stimulation has been described as intense and usually greater in those patients who are weaned successfully, with a consequent increase in systemic catecholamine levels [22]. The imbalance between the capacity and loading conditions of the respiratory and cardiovascular system during the weaning process could entail major cardiopulmonary stress, especially in patients with limited baseline conditions, causing weaning failure, and triggering the somatic stress response and a potential increase in IL-6 $[2,5]$.

In our study, the increase of serum IL-6 levels was higher in SBT failure compared with success, especially in the COPD population. These results may be attributed to the fact that COPD patients present less favourable reserve conditions for coping with cardiopulmonary stress during the SBT than non-COPD patients $[6,10,23]$, which explains the high rate of weaning failure typically described in COPD [24]. From our results, it appears that only COPD patients without excessive stress (no changes in IL-6) can achieve SBT success. In contrast, IL-6 levels were not influenced by the performance of a SBT in non-COPD patients, possibly due to the fact that they presented a better reserve for coping with the SBT stress than COPD patients. However, this hypothesis should be tested in further studies.

The post-stress response observed at $24 \mathrm{~h}$ after the trial was more consonant with the initial hypothesis. The majority of the patients, except those who developed respiratory failure after extubation, showed a decrease in IL-6 at 24 h, which was highly significant in the event of SBT failure (possibly due to the relative resting of the cardiorespiratory system after the increased stress of a failed SBT) and in weaning success (showing the relative clinical stability of patients after extubation). This IL-6 response, particularly in SBT failure, was similar to the decline observed in the post-stress period reported in the stress model of acute exercise [14].

Apart from IL-6, we did not obtain significant results from the analysis of the remaining mediators. These results suggest the absence of a marked inflammatory response during a 30-min SBT, despite the increase in IL-6, which is similar to the "exercise" condition (stress secondary to a physical exercise) and contrasts with sepsis, where several inflammatory mediators are increased [14]. In fact, the SBT is physiologically closer to exercise than other types of stress [25]. It has even been hypothesised that IL- 6 could exert inhibitory effects in TNF- $\alpha$ and IL-1 $\beta$ during acute exercise, playing an anti-inflammatory role [14]. Another explanation for our results could be that the remaining inflammatory markers may increase just after the
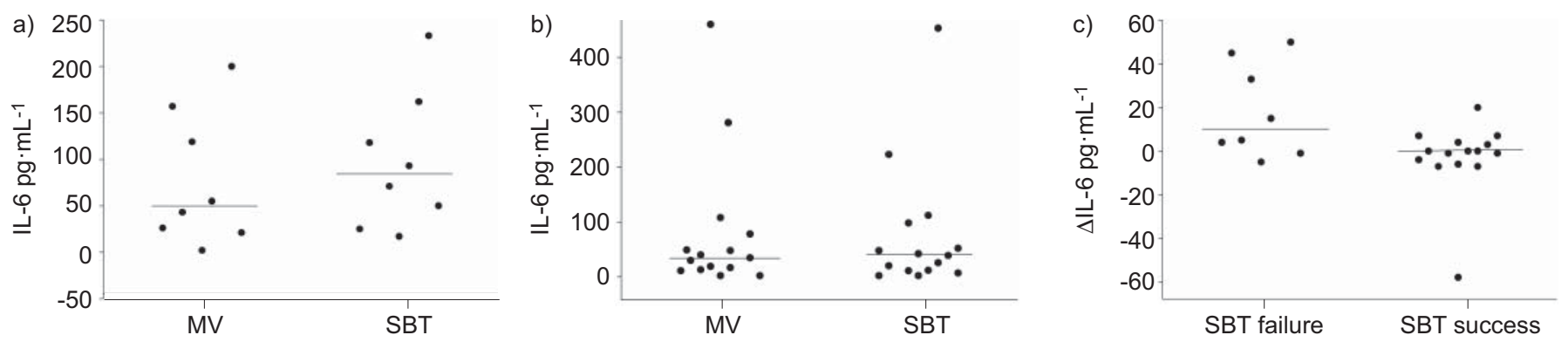

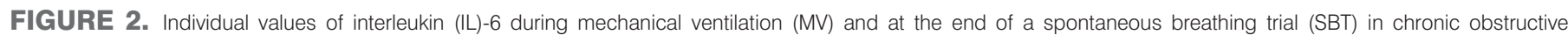

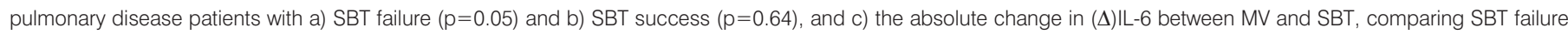
and success $(p=0.03)$. - : median. 

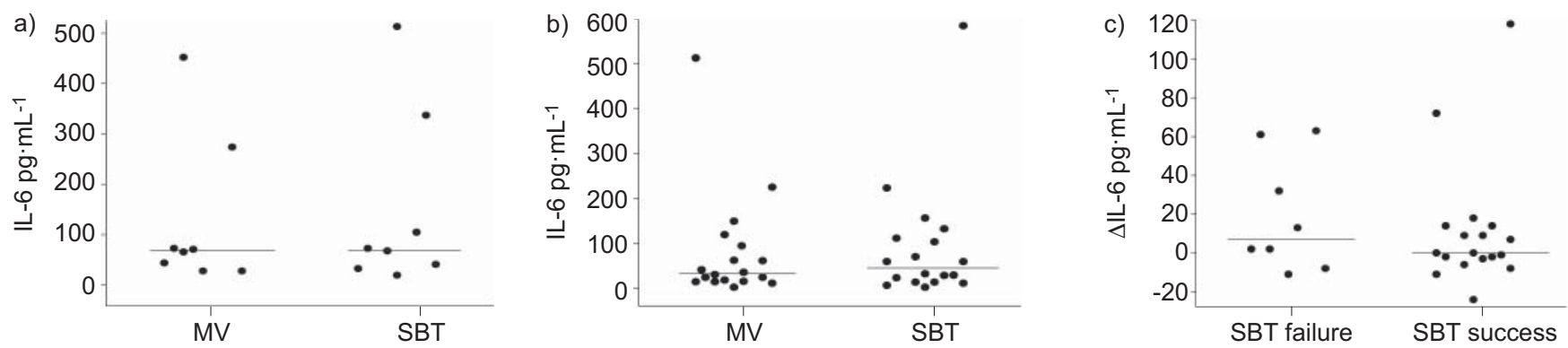

FIGURE 3. Individual values of interleukin (IL)-6 during mechanical ventilation (MV) and at the end of a spontaneous breathing trial (SBT) in patients without chronic obstructive pulmonary disease, with a) SBT failure $(p=0.12)$ and b) SBT success $(p=0.25)$, and c) the absolute change in $(\Delta) / L-6$ between MV and SBT, comparing SBT failure and success $(p=0.49)$. - : median

SBT, because IL-6 appearance in the circulation is by far the most marked and its increase precedes the other cytokines in stress secondary to exercise [14].

In our opinion, the current study may open novel possibilities in weaning research. Physiologically, the role of IL-6 in the weaning process must be elucidated. Future research must explore the connection of IL-6 with the hypothalamic-pituitaryadrenal axis and sympathetic system during the SBT and the hypothetical metabolic and hormonal implications of the rise in IL-6. As has been previously described, there is an increase in several hormonal modulators associated with stress during a SBT [21, 22]. IL-6 is a major modulator of the different systems triggered during a stress response [5], so we have hypothesised that IL-6 could play a key role in the stress response associated with SBT, especially in COPD. The potential clinical consequences of an increased response of IL-6 and other stress modulators during the SBT remain unknown, but it would be of interest to determine the threshold at which an increased stress response during a SBT could have deleterious systemic effects. Actually, systemic IL-6 overproduction is associated with different actions in haematological, immunological, hepatic, neurological, cardiac and endocrine systems [12]. This hypothesis may well have important clinical implications in different prognostic outcomes. In fact, IL-6 is associated with mortality in other stress conditions, such as acute respiratory distress syndrome [26] or sepsis [27].

Certain limitations of the study must be considered. First, the results obtained at $24 \mathrm{~h}$ must be interpreted with caution, as external factors other than weaning could have influenced the final values and this might prevent appropriate interpretation of the data. Secondly, another limitation of our study is the limited duration of SBT (30 min). Patients usually fail a SBT within the first $20 \mathrm{~min}$, so the success rate of a 30-min SBT is very similar to a 120 -min trial $[28,29]$. Nevertheless, it is possible that during longer SBTs, the increase in IL- 6 could be higher, due to the progressive increase in inspiratory effort during the trial [30]. Thirdly, the data obtained from the COPD and non-COPD populations were obtained from an analysis of subgroups, so the results must be viewed with caution and stand to be confirmed in specific studies addressed to these populations.

In conclusion, our results provide the first evidence that systemic IL-6 increases during the course of a 30-min, failed SBT, especially in COPD patients. No significant changes were observed in the remaining inflammatory mediators studied.
Future research could serve to explore the association of IL-6 levels and the mechanisms of weaning failure in COPD patients, and the possible association with prognostic outcomes.

\section{SUPPORT STATEMENT}

This work was supported by Ministerio de Sanidad y Consumo (FISPI040929 and CibeRes-ISCiii-CB06/06/0028) and Ministerio de Ciencia y Tecnología (SAF2005-0110).

\section{STATEMENT OF INTEREST}

None declared.

\section{ACKNOWLEDGEMENTS}

The authors wish to thank M. Valencia (Hospital Sagrat Cor, Barcelona, Spain), X. Filella and S. Blanco for their technical assistance, and R. Marshall (all Hospital Clínic, Barcelona) for his editing aid. The authors also thank the respiratory therapy and nursing staff, and the attending physicians of the intensive care unit, for cooperation in this study.

\section{REFERENCES}

1 Ely EW, Baker AM, Dunagan DP, et al. Effect on the duration of mechanical ventilation of identifying patients capable of breathing spontaneously. N Engl J Med 1996; 335: 1864-1869.

2 Boles JM, Bion J, Connors A, et al. Weaning from mechanical ventilation. Eur Respir J 2007; 29: 1033-1056.

3 Esteban A, Frutos F, Tobin MJ, et al. A comparison of four methods of weaning patients from mechanical ventilation. $N$ Engl J Med 1995; 323: 345-350.

4 Girard TD, Kress JP, Fuchs BD, et al. Efficacy and safety of a paired sedation and ventilator weaning protocol for mechanically ventilated patients in intensive care (Awakening and Breathing Controlled trial): a randomised controlled trial. Lancet 2008; 371: 126-134.

5 Mastorakos G, Ilias I. Interleukin-6 - a cytokine and/or a major modulator of the response to somatic stress. Ann NY Acad Sci 2006; 1088: 373-381.

6 Jubran A, Tobin MJ. Pathophysiologic basis of acute respiratory distress in patients who fail a trial of weaning from mechanical ventilation. Am J Respir Crit Care Med 1997; 155: 906-915.

7 Jubran A, Mathru M, Dries D, et al. Continuous recordings of mixed venous oxygen saturation during weaning from mechanical ventilation and the ramifications thereof. Am J Respir Crit Care Med 1998; 158: 1763-1769.

8 De Backer D, El Haddad P, Preiser JC, et al. Hemodynamic responses to successful weaning from mechanical ventilation after cardiovascular surgery. Intensive Care Med 2000; 26: 1201-1206. 
9 Richard C, Teboul JL, Archambaud F, et al. Left ventricular function during weaning of patients with chronic obstructive pulmonary disease. Intensive Care Med 1994; 20: 181-186.

10 Lemaire F, Teboul J, Cinotti L, et al. Acute left ventricular dysfunction during unsuccessful weaning from mechanical ventilation. Anesthesiology 1988; 69: 171-179.

11 Mastorakos G, Pavlatou M. Exercise as a stress model and the interplay between the hypothalamus-pituitary-adrenal and the hypothalamus-pituitary-thyroid axes. Horm Metab Res 2005; 37: 577-584.

12 Papanicolaou DA, Wilder RL, Manolagas SC, et al. The pathophysiologic roles of interleukin-6 in human disease. Ann Intern Med 1998; 128: 127-137.

13 Ostrowski K, Schjerling P, Pedersen BK. Physical activity and plasma interleukin-6 in humans-effect of intensity of exercise. Eur J Appl Physiol 2000; 83: 512-515.

14 Petersen AM, Pedersen BK. The role of IL-6 in mediating the antiinflammatory effects of exercise. J Physiol Pharmacol 2006; 57: Suppl. 10, 43-51.

15 Ferrer M, Esquinas A, Arancibia F, et al. Noninvasive ventilation during persistent weaning failure. A randomized controlled trial. Am J Respir Crit Care Med 2003; 168: 70-76.

16 Ferrer M, Valencia M, Nicolas JM, et al. Early noninvasive ventilation averts extubation failure in patients at risk: a randomized trial. Am J Respir Crit Care Med 2006; 173: 164-170.

17 Truwit JD, Marini JJ. Validation of a technique to assess maximal inspiratory pressure in poorly cooperative patients. Chest 1992; 102: 1216-1219.

18 Epstein SK, Ciubotaru RL. Independent effects of etiology of failure and time of reintubation on outcome for patients failing extubation. Am J Respir Crit Care Med 1998; 158: 489-493.

19 Brochard L, Rauss A, Benito S, et al. Comparison of three methods of gradual withdrawal from ventilatory support during weaning from mechanical ventilation. Am J Respir Crit Care Med 1994; 150: 896-903.
20 Sellares J, Ferrer M, Farré R, et al. Serum inflammatory biomarkers in weaning from mechanical ventilation. Am J Respir Crit Care Med 2008; 177: Suppl., A378.

21 Koksal GM, Sayilgan C, Sen O, et al. The effects of different weaning modes on the endocrine stress response. Crit Care 2004; 8: R31-R34.

22 Kennedy SK, Weintraub RM, Skillman JJ. Cardiorespiratory and sympathoadrenal responses during controlled ventilation. Surgery 1977; 82: 233-240.

23 Grasso S, Leone A, De Michele M, et al. Use of N-terminal probrain natriuretic peptide to detect acute cardiac dysfunction during weaning failure in difficult-to-wean patients with chronic obstructive pulmonary disease. Crit Care Med 2007; 35: 96-105.

24 Vallverdú I, Calaf N, Subirana M, et al. Clinical characteristics, respiratory functional parameters, and outcome of a two-hour Tpiece trial in patients weaning from mechanical ventilation. Am J Respir Crit Care Med 1998; 158: 1855-1862.

25 Pinsky MR. Breathing as exercise: the cardiovascular response to weaning from mechanical ventilation. Intensive Care Med 2000; 26: 1164-1166.

26 Parsons PE, Eisner MD, Thompson BT, et al. Lower tidal volume ventilation and plasma cytokine markers of inflammation in patients with acute lung injury. Crit Care Med 2005; 33: 1-6.

27 Song M, Kellum JA. Interleukin-6. Crit Care Med 2005; 33: Suppl., S463-S465.

28 Esteban A, Alía I, Tobin MJ, et al. Effect of spontaneous breathing trial duration on outcome of attempts to discontinue mechanical ventilation. Am J Respir Crit Care Med 1999; 159: 512-518.

29 Perren A, Domenighetti G, Mauri S, et al. Protocol-directed weaning from mechanical ventilation: clinical outcome in patients randomized for a $30-\mathrm{min}$ or 120 -min trial with pressure support ventilation. Intensive Care Med 2002; 28: 1058-1063.

30 Jubran A, Grant BJB, Laghi F, et al. Weaning prediction esophageal pressure monitoring complements readiness testing. Am J Respir Crit Care Med 2005; 171: 1252-1259. 